The Astrophysical JournaL, 690: L97-L100, 2009 JANUARY 10

Preprint typeset using LTEX style emulateapj v. 08/22/09

\title{
ON THE ORIGIN OF SPECTRAL STATES IN ACCRETING BLACK HOLES
}

\author{
JURI POUTANEN ${ }^{1}$ AND INDREK VURM ${ }^{1,2}$ \\ Received 2008 July 19; accepted 2008 November 20; published 2008 December 15
}

\begin{abstract}
The origin of dramatically different electron distributions responsible for Comptonization in black hole Xray binaries (BHBs) in their various states is discussed. We solve the coupled kinetic equations for photons and electrons without approximations on the relevant cross-sections accounting for Compton scattering, synchrotron radiation, and Coulomb collisions. In the absence of external soft photons, the electrons are efficiently thermalized by synchrotron self-absorption and Coulomb scattering even for pure nonthermal electron injection. The resulting quasi-thermal synchrotron self-Compton spectra have very stable slopes and electron temperatures similar to the hard states of BHBs. The hard spectral slopes observed in the X-rays, the cutoff at $100 \mathrm{keV}$, and the $\mathrm{MeV}$ tail together require low magnetic fields ruling out the magnetic dissipation mechanism. The motion of the accretion disk toward the black hole results in larger Compton cooling and lower equilibrium electron temperature. Our self-consistent simulations show that in this case both electron and photon distributions attain a power-law-dominated shape similar to what is observed in the soft state. The electron distribution in the Cyg X-1 soft state might require a strong magnetic field, being consistent with the magnetically dominated corona.

Subject headings: accretion, accretion disks - black hole physics - gamma-rays: theory - methods: numerical - radiation mechanisms: nonthermal - X-rays: binaries
\end{abstract}

\section{INTRODUCTION}

The physical processes giving rise to the X-ray/gamma-ray emission of accreting black-hole binaries (BHBs) have been a matter of debates over the last four decades. The hard-state spectra, showing a strong cut-off around $100 \mathrm{keV}$, are well described by thermal Comptonization (e.g. Poutanen 1998; Zdziarski \& Gierliński 2004), while a weak MeV tail requires the presence of nonthermal particles (McConnell et al. 1994; Ling et al. 1997). The origin of seed soft photons for Comptonization is, however, much less clear. An apparent correlation between the spectral slope and the amount of Compton reflection (Zdziarski et al. 1999) argues in favor of the accretion disk, while the observed optical/X-ray correlation (Motch et al. 1982; Kanbach et al. 2001) leans toward the synchrotron hypothesis (e.g. Fabian et al. 1982; Wardziński $\&$ Zdziarski 2001). Interesting questions are then: what stabilizes the X-ray spectral slope at $\alpha \sim 0.6-0.8$, and what fixes the temperature of the emitting plasma at $k T_{\mathrm{e}} \sim 50-100 \mathrm{keV}$ (Zdziarski et al. 1997; Poutanen 1998; Zdziarski \& Gierliński 2004)? Do the feedback from the cool accretion disk and the thermostatic properties of electron-positron pairs (Haardt \& Maraschi 1993; Haardt et al. 1994; Stern et al. 1995; Malzac et al. 2001) play a role here? Or does the cooling by synchrotron radiation (Narayan \& Yi 1995) act as a stabilizer?

In the soft state, BHB spectra are dominated by thermal disk emission of temperature $k T_{\mathrm{BB}} \sim 0.4-1.5 \mathrm{keV}$. At higher energies the spectrum is power-law-like and shows no signatures of the cut-off (Grove et al. 1998) extending possibly up to $10 \mathrm{MeV}$ (McConnell et al. 2002). This emission is well described by Comptonization in almost purely nonthermal plasmas (Poutanen \& Coppi 1998; Gierliński et al. 1999; Coppi 1999; Zdziarski et al. 2001; Zdziarski \& Gierliński 2004). We can then ask why the electrons are nearly thermal in the hard

\footnotetext{
${ }^{1}$ Astronomy Division, Department of Physical Sciences, P.O.Box 3000, 90014 University of Oulu, Finland; juri.poutanen@oulu.fi, indrek.vurm@oulu.fi

${ }^{2}$ Tartu Observatory, 61602 Tõravere, Tartumaa, Estonia; vurm@ut.ee
}

state, and what causes such a dramatic change in the electron distribution when transition to the soft state happens. Poutanen \& Coppi (1998) proposed that the two states are distinguished by the way the energy is supplied to the electrons: by thermal heating, dominating during the hard state, and by nonthermal acceleration, operating in the soft state. However, their treatment of Coulomb collisions (using EQPAIR code by Coppi 1992, 1999) was approximate, and they have neglected the effect of synchrotron boiler, involving the emission and absorption of synchrotron photons, which can act as an efficient particle thermalizer (Ghisellini et al. 1988).

Ghisellini et al. (1998) studied for the first time the combined effect of the synchrotron boiler and Compton cooling on the electron distribution and photon spectra (but neglected Coulomb scattering). They considered a two-phase corona model (Haardt \& Maraschi 1993; Haardt et al. 1994; Stern et al. 1995), where half of the high-energy radiation was assumed to be reprocessed by the disk to soft photons. As the actual geometry of the emitting region is not known, we start from pure synchrotron self-Compton models (i.e. with no external soft photons) and compute self-consistently the electron (positron) and photon distributions. We then investigate how the additional soft photons (e.g., associated with the inner radius of the cool accretion disk) affect the equilibrium distributions and compare the results of simulations with the data on Cyg X-1. The preliminary results of this study were presented by Vurm \& Poutanen (2008a).

\section{MODEL SETUP}

We consider a black hole of mass $10 M_{\odot}$, typical for stellar-mass BHBs. We assume that the inner accretion flow is hot and almost spherical, corresponding to the advectiondominated (Narayan \& Yi 1995; Abramowicz et al. 1995; Esin et al. 1997) or to the recently discovered luminous hot accretion flow solutions (Yuan 2003; Yuan \& Zdziarski 2004). One expects that most of the gravitational energy release happens within about $R=10 R_{\mathrm{S}}=3 \times 10^{7} \mathrm{~cm}$ (where $R_{\mathrm{S}}=2 G M / c^{2}$ is the Schwarzschild radius) from the black 
hole, and we thus fix the size of the active region at this value in most of the simulations. The released energy needs to be transferred to electrons via, e.g., Coulomb collisions with hot protons, collective plasma effects, magnetic reconnection, or shocks. We assume that the energy transfer to the electrons is given by a power-law-injection function $d N_{\mathrm{e}} /(d t d \gamma) \propto \gamma^{-\Gamma_{\text {inj }}}$ extending in the Lorentz factor from $\gamma=1$ to $10^{3}$. To keep the Thomson optical depth of the electrons associated with protons $\tau_{\mathrm{p}}$ constant, ${ }^{3}$ the same number of electrons from the equilibrium distribution is removed from the system. In this case, the net power is $L_{\text {inj }}=\frac{4 \pi}{3} R^{3} \dot{N}_{\mathrm{e}}\left(\langle\gamma\rangle_{\text {inj }}-\langle\gamma\rangle_{\text {eq }}\right) m_{\mathrm{e}} c^{2}$, where $\langle\gamma\rangle_{\text {inj }}$ and $\langle\gamma\rangle_{\text {eq }}$ are the mean Lorentz factors of the injection function and of the equilibrium distribution, respectively, and $\dot{N}_{\mathrm{e}}$ is uniquely determined by the model parameters and $\langle\gamma\rangle_{\text {eq. }}$.

The injected electrons are cooled by synchrotron emission and Compton scattering at timescales much shorter than the accretion time. The synchrotron radiation is strongly selfabsorbed up to hundreds of harmonics, and therefore the cooling depends strongly on the high-energy tail of the electron distribution (see e.g. Wardziński \& Zdziarski 2001). The importance of synchrotron processes is determined by the ratio $\eta_{\mathrm{B}}=U_{\mathrm{B}} R^{2} c / L_{\mathrm{inj}}$, where $U_{\mathrm{B}}=B^{2} /(8 \pi)$ is the magnetic energy density and $L_{\mathrm{inj}} \approx \frac{4 \pi}{3} R^{2} c U_{\mathrm{rad}}$ (so that $\eta_{\mathrm{B}} \approx 3 / 4 \pi \approx 0.25$ corresponds to an equipartition of the magnetic and radiation energy densities, $U_{\mathrm{B}}=U_{\text {rad }}$ ). The seed photons for Compton upscattering can be provided by the synchrotron as well as by the external sources, the cool accretion disk being the most natural one. The external soft photons are modeled as a blackbody of temperature $T_{\mathrm{BB}}$ determined from the Stefan-Boltzmann law $L_{\text {disk }}=4 \pi R^{2} \sigma_{\mathrm{SB}} T_{\mathrm{BB}}^{4}$. The cooling by external photons depends on the ratio $f=L_{\text {disk }} / L_{\text {inj }}$. The total escaping photon luminosity is $L=L_{\text {disk }}+L_{\text {inj }}=(1+f) L_{\text {inj }}$.

To model self-consistently particle and photon distributions, we solve numerically a set of coupled, time-dependent kinetic equations for photons, electrons, and positrons describing Compton scattering, cyclo-synchrotron emission and absorption, electron-electron Coulomb (Møller) scattering as well as pair production and annihilation. Our calculations are done in a simple one-zone geometry with a tangled magnetic field and isotropic particle and photon distributions. The escape probability formalism is used to simulate photon escape from the region. The detailed description of the code and its extensive testing are given in Vurm \& Poutanen (2008b).

\section{SYNCHROTRON SELF-COMPTON MODELS}

We first assume that the cool disk is sufficiently far away and does not supply any seed soft photons to the inner hot flow. Thus we consider pure synchrotron self-Compton (SSC) models $(f=0)$. We choose the fiducial parameter set $L=$ $L_{\text {inj }}=10^{37} \mathrm{erg} \mathrm{s}^{-1}$ and $\tau_{\mathrm{p}}=1.5$ (typical for the hard state of BHBs, Zdziarski et al. 1997), $R=3 \times 10^{7} \mathrm{~cm}, \Gamma_{\text {inj }}=3(\mathrm{ad}$ hoc), and $\eta_{\mathrm{B}}=1$. The equilibrium electron distribution consists of a Maxwellian part with $k T_{\mathrm{e}}=66 \mathrm{keV}$ and a power-lawlike tail with the slope modified by cooling $\Gamma_{\mathrm{e}}=\Gamma_{\mathrm{inj}}+1=4$ (where $d N_{\mathrm{e}} / d \gamma \propto \gamma^{-\Gamma_{\mathrm{e}}}$; see the solid curve in Fig. 1a'). The synchrotron emission is strongly self-absorbed with only the nonthermal tail above $\gamma \gtrsim 20$ contributing to emission above the self-absorption energy at $\gtrsim 10 \mathrm{eV}$. As the amount of seed

\footnotetext{
3 The total optical depth might be larger due to the produced pairs, but for parameters considered here, the amount of pairs is negligible.
}

TABLE 1

RESULTS OF SIMULATIONS

\begin{tabular}{cccr}
\hline \multicolumn{2}{c}{$\mathrm{P}^{\mathrm{a}}$} & $\alpha^{\mathrm{b}}$ & $\begin{array}{r}T_{\mathrm{e}}^{\mathrm{c}} \\
(\mathrm{keV})\end{array}$ \\
\hline \multicolumn{2}{c}{ Fiducial $^{\mathrm{d}}$} & 0.89 & 66 \\
\hline$\Gamma_{\text {inj }}$ & 2 & 1.07 & 27 \\
& 4 & 0.73 & 90 \\
\hline$\eta_{\mathrm{B}}$ & 0.1 & 0.75 & 77 \\
& 10 & 0.98 & 64 \\
\hline$\tau_{\mathrm{p}}$ & 0.15 & 1.12 & 160 \\
& 15 & 0.58 & 4 \\
\hline$f$ & 0.1 & 0.97 & 61 \\
& 0.3 & 1.13 & 49 \\
& 1 & 1.61 & 31 \\
& 3 & 2.46 & 16 \\
\hline
\end{tabular}

a Varying parameter and its value.

${ }^{b}$ Photon spectral index in the $2-10 \mathrm{keV}$ range.

${ }^{\mathrm{c}}$ Temperature of the Maxwellian part of the electron distribution.

${ }^{\mathrm{d}}$ Fiducial set of parameters $L=10^{37} \mathrm{erg} \mathrm{s}^{-1}, R=3 \times 10^{7} \mathrm{~cm}, f=0$, $\Gamma_{\text {inj }}=3, \tau_{\mathrm{p}}=1.5, \eta_{\mathrm{B}}=1$.

soft (synchrotron) photons is low, the Comptonization spectrum (produced predominantly by the thermal electron population) is hard with the photon energy index $\alpha \approx 0.9$ and a cut-off at $\sim 100 \mathrm{keV}$, which is similar to the hard state of BHBs. A tail produced by single-Compton scattering off the power-law electron tail is clearly visible above MeV.

Variation of the slope of the injected electrons leads to a large change in the tail of the electron distribution and dramatic difference in the synchrotron emission. A soft electron injection with $\Gamma_{\text {inj }} \gtrsim 4$ leads to efficient thermalization and a small amount of soft photons resulting in rather hard radiation spectra, with the photon energy index $\alpha \lesssim 0.7$ (see dashed curves in Fig. 1a). A hard injection gives more power to the nonthermal tail and more seed photons for Comptonization (see also Ghisellini et al. 1998; Wardziński \& Zdziarski 2001), which causes a drop in the electron temperature (see the dotted curves in Fig. 1a). A strong 'bump' also develops in the tail of the electron distribution at $\gamma \sim 3$. The synchrotron emission produced by these electrons is still strongly self-absorbed, while the energy losses and gains stay close to each other for an extended energy interval (Katarzyński et al. 2006). In this regime, the ratio of synchrotron heating and cooling rates for a power-law distribution of relativistic electrons is $\dot{\gamma}_{\mathrm{h}} / \dot{\gamma}_{\mathrm{c}} \approx 5 /\left(\Gamma_{\mathrm{e}}+2\right) .{ }^{4}$ Observe that for $\Gamma_{\mathrm{e}}=3$ (i.e. for $\Gamma_{\text {inj }}=2$ ) the heating and cooling rates are balanced, however, such an equilibrium is unstable (Rees 1967). The Comptonized spectrum for hard injection $\Gamma_{i n j}=2$ (see Fig. 1a) is much softer than the hard-state spectra of BHBs, even without any contribution to the cooling from the disk, strongly constraining the electron injection mechanism in BHBs.

The efficiency of synchrotron cooling depends on the magnetic field strength parameterized here via magnetization $\eta_{\mathrm{B}}$. At small $\eta_{\mathrm{B}}$ (see Fig. 1b'), synchrotron is inefficient and cooling is dominated by thermal Comptonization. A higher normalization of the power-law part of the equilibrium electron distribution leads to a stronger $\mathrm{MeV}$ tail. For $\eta_{\mathrm{B}} \lesssim 1$ (and $\Gamma_{\text {inj }}>3$ ), the thermal Comptonization spectrum is very stable with $\alpha \sim 0.7-0.9$. At large $\eta_{\mathrm{B}}>1$, the synchrotron thermalization operates more efficiently and the thermal part of the

\footnotetext{
4 This expression can be derived by employing the delta-function approximation for synchrotron emissivity to calculate the source function and using it in the expression for heating by self-absorption (see e.g. Ghisellini et al. 1988; Katarzyński et al. 2006).
} 

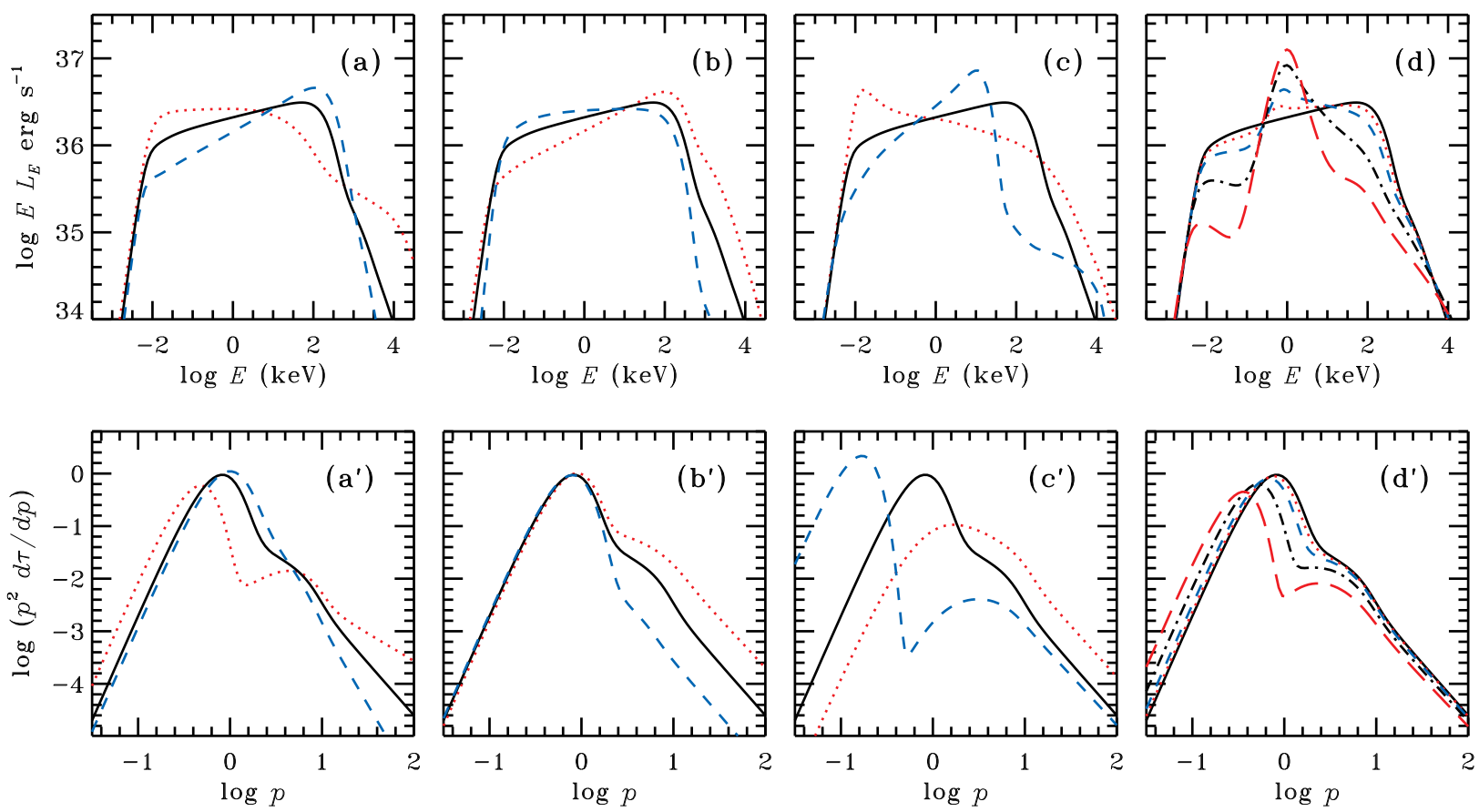

FIG. 1.- Equilibrium photon spectra (upper panels) and electron distributions (lower panels) $p^{2} d \tau / d p$ (i.e., momentum per log $p$, where $\tau$ is the Thomson optical depth and $p=\sqrt{\gamma^{2}-1}$ is the dimensionless electron momentum). The results for the fiducial parameter set $L=L_{\mathrm{inj}}=10^{37} \mathrm{erg} \mathrm{s} \mathrm{s}^{-1}, \Gamma_{\mathrm{inj}}=3$, $\tau_{\mathrm{p}}=1.5, \eta_{\mathrm{B}}=1, f=0$, are shown by solid curves in all panels. (a-a') Dependence on the electron injection slopes: $\Gamma_{\mathrm{inj}}=2$ (dotted curve), 3 (solid) and 4 (dashed). (b-b') Dependence on magnetization $\eta_{\mathrm{B}}=0.1$ (dotted), 1 (solid), 10 (dashed). (c-c') Dependence on the optical depth $\tau_{\mathrm{p}}=0.15$ (dotted), 1.5 (solid), 15 (dashed). (d-d') Dependence on the ratio of the external disk photons to the injected power $f=0,0.1,0.3,1,3$ (solid, dotted, dashed, dot-dashed, long-dashed curves, respectively) for constant total luminosity $L$. The electron temperatures and photon spectral indices are given in Table 1.

distribution persists to higher energies. The increasing $B$ field compensates for the decrease in the power-law tail leading to a higher synchrotron emission, which results in softening of the Comptonized spectrum.

Consider now variations of $\tau_{\mathrm{p}}$ for the fixed $L_{\mathrm{inj}}$. At high $\tau_{\mathrm{p}}$, the equilibrium electron temperature drops, leaving fewer energetic electrons for synchrotron emission and, therefore, reducing the number of seed photons for Comptonization (Fig. 1c). This, in turn, results in the harder photon spectra produced by saturated Comptonization (by thermal electrons) and a weak high-energy tail (produced by nonthermal electrons), very similar to the ultrasoft spectra of BHBs (see figs. 8 and 9 in Zdziarski \& Gierliński 2004). At smaller $\tau_{\mathrm{p}}$, the higher electron temperature leads to a stronger synchrotron cooling and to a lower Comptonized luminosity and, therefore, softer Comptonized spectra.

Let us now apply the developed model to the hard state of Cyg X-1. The MeV tail observed there with $\alpha_{\mathrm{MeV}} \approx 2$ (McConnell et al. 2002) constrains the injection slope to be $\Gamma_{\text {inj }}<2 \alpha_{\mathrm{MeV}}=4$. Then the hard X-ray spectra with $\alpha \lesssim 0.7$ and a high-energy cutoff at $\sim 100 \mathrm{keV}$ (see Fig. 2) require $\tau_{\mathrm{p}} \sim 1$ and low $\eta_{\mathrm{B}} \lesssim 0.1$ (see also Wardziński \& Zdziarski 2001; McConnell et al. 2002). Any additional soft photons from the disk will make the spectrum softer, reducing $\eta_{\mathrm{B}}$ even more. The low magnetic field rules out magnetic reconnection as the energy dissipation mechanism. This also implies that electrons cannot be thermalized by the synchrotron selfabsorption. On the other hand, if the size of the active region is $R \sim 60 R_{\mathrm{S}}$, Coulomb scattering becomes important (as its influence grows linearly with size for constant $L$, see, e.g., Coppi 1999; Svensson 1999), and it can thermalize electrons at a rather high temperature of $k T_{\mathrm{e}} \sim 100 \mathrm{keV}$ as observed in Cyg X-1 (Gierlinski et al. 1997; Poutanen 1998).
We reiterate that the whole spectrum here is produced by the SSC mechanism. ${ }^{5}$ Its thermostatic properties fix the electron temperature at $50-100 \mathrm{keV}$ (for $\tau_{\mathrm{p}} \sim 1$ ) and stabilize the spectral slope at $\alpha \sim 0.7-0.9$. The feedback from the disk (Haardt \& Maraschi 1993; Haardt et al. 1994; Stern et al. 1995; Malzac et al. 2001) does not seem to be needed.

\section{SPECTRAL TRANSITIONS AND THE ROLE OF DISK PHOTONS}

The spectral transitions observed in BHBs are most probably accompanied by a change in the geometry of the accretion disk. The cool outer disk moves toward the central black hole causing an increasing flux of the soft photons to the central hot flow (Esin et al. 1997; Poutanen et al. 1997; Poutanen \& Coppi 1998), which we simulate here by increasing $f$ (see Fig. 1d). Higher soft photon flux leads to faster Compton cooling and lower equilibrium electron temperature, making the nonthermal part more pronounced. The resulting photon distribution changes from the hard, thermal Comptonization dominated, spectrum to the one dominated by the disk blackbody, with a nonthermal tail extending to tens of $\mathrm{MeV}$, which becomes harder at higher $f$. The spectral changes triggered by varying $f$ are similar to the one observed in Cyg X-1 (see Fig. 2). A detailed comparison with Cyg X-1 spectra shows, however, that other parameters change too.

Compared to the hard state, the soft state corresponds to a higher total luminosity. The $\mathrm{MeV}$ tail is harder $\alpha_{\mathrm{MeV}} \approx 1.6$ (McConnell et al. 2002), and therefore $\Gamma_{\mathrm{inj}}<3.2$. If the tail of the black-body at 3-10 keV (see Fig. 2) is produced in the same emission region, it requires a rather hot thermal popu-

5 ADAF-based models also consider SSC as the main cooling mechanism; see Narayan et al. (1998) for the review. 


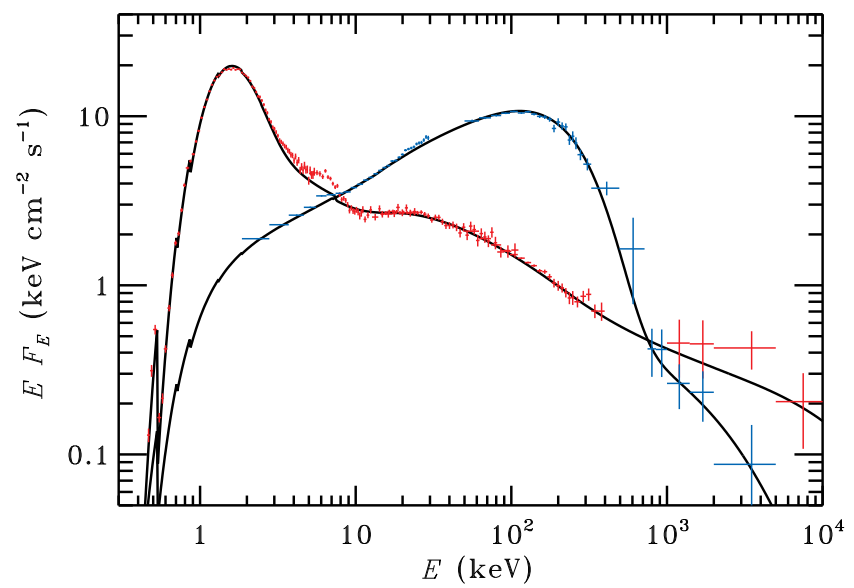

FIG. 2.- Spectral states of Cyg X-1. Crosses show the unfolded spectral data presented by Zdziarski et al. (2002). The model spectra with interstellar absorption (described by the column density $N_{\mathrm{H}}$ ) and Compton reflection (described by the solid angle $\Omega$ and ionization parameter $\xi$, Magdziarz \& Zdziarski 1995) taken into account are shown by the solid curves. The parameters for the hard-state model are: $L=2.7 \times 10^{37} \mathrm{erg} \mathrm{s}^{-1}, R=1.8 \times 10^{8}$ $\mathrm{cm}, f=0, \Gamma_{\mathrm{inj}}=3.8, \tau_{\mathrm{p}}=2.5, \eta_{\mathrm{B}}=0.083, N_{\mathrm{H}}=3 \times 10^{21} \mathrm{~cm}^{-2}$, $\Omega /(2 \pi)=0.2, \xi=0$. The soft state can be described by $L=4.85 \times 10^{37}$ $\mathrm{erg} \mathrm{s}^{-1}, R=3.85 \times 10^{7} \mathrm{~cm}, f=2.13, \Gamma_{\mathrm{inj}}=2.2, \tau_{\mathrm{p}}=0.3, \eta_{\mathrm{B}}=1.5$, $N_{\mathrm{H}}=5 \times 10^{21} \mathrm{~cm}^{-2}, \Omega /(2 \pi)=0.7, \xi=100$.

lation of electrons, which needs high $\eta_{\mathrm{B}}$ for the synchrotron thermalization to operate (because Coulomb thermalization is not efficient under the conditions of strong Compton cooling). This would be consistent with the magnetically dominated emission region. Alternatively, there may be additional heating mechanisms operating. Also the tail might be a result of Comptonization in the hot ionized skin of the disk, not directly related to the emission we discuss here, but this interpretation might not be easily reconciled with the fact that the disk is stable, while the tail varies (Churazov et al. 2001). While the dramatic changes in the electron and photon distributions between the states are mainly caused by variations of the disk luminosity, it is obvious that other parameters do change during the transition. We stress that none of the presented models requires any additional thermal heating, which is different from the models of Poutanen \& Coppi (1998) and Gierliński et al. (1999).

\section{CONCLUSIONS}

The hard state of BHBs can well be described by the quasithermal SSC mechanism. The feedback from the cool disk is not needed to stabilize the spectral slope and the electron temperature. Electrons can be injected to the active region with the power-law spectrum, but Coulomb scattering and synchrotron self-absorption thermalize them efficiently. This reduces the need for mysterious 'thermal heating' that was invoked previously to explain thermal Comptonization spectra of BHBs. The MeV tail together with the hard X-ray spectra of BHBs with photon indices $\alpha \lesssim 0.7$ and a cutoff at $100 \mathrm{keV}$ require rather low magnetization $\eta_{\mathrm{B}}<0.1$ and a large size of $R \gtrsim 60 R_{\mathrm{S}}$. In that case, magnetic reconnection can be ruled out as a source of energy.

At high optical depth of the emitting region $\tau_{\mathrm{p}} \gtrsim 10$, in the absence of disk radiation, the spectrum is close to saturated Comptonization, peaking at a few keV. This Wien-type spectrum might be associated with the ultrasoft state of BHBs. At low $\tau_{\mathrm{p}}$, the electrons are hotter and the spectra are softer due to the efficient synchrotron cooling.

A behavior similar to what is observed during the spectral state transitions in BHBs can be reproduced by varying the ratio of injected soft luminosity and the power dissipated in the hot phase, which could be caused by varying the radius of the inner cool disk. The increasing Compton cooling causes dramatic changes in the electron distribution from almost purely thermal to nearly nonthermal. The photon distribution also changes from quasi-thermal SSC to the nonthermal Comptonization of the disk photons. In the soft state of Cyg X1 , a strong magnetic field can thermalize electrons at sufficiently high temperature, which is consistent with a magnetically dominated corona being responsible for the high-energy emission.

We are grateful to the anonymous referee and Andrzej Zdziarski for a number of useful comments. This study was supported by the CIMO grant TM-06-4630 and the Academy of Finland grants 110792 and 122055.

\section{REFERENCES}

Abramowicz, M. A., Chen, X., Kato, S., Lasota, J.-P., \& Regev, O. 1995, ApJ, 438, L37

Churazov, E., Gilfanov, M., \& Revnivtsev, M. 2001, MNRAS, 321, 759 Coppi, P. S. 1992, MNRAS, 258, 657

Coppi, P. S. 1999, in ASP Conf. Ser. 161, High Energy Processes in Accreting Black Holes, ed. J. Poutanen \& R. Svensson (San Francisco: ASP), 375

Esin, A. A., McClintock, J. E., \& Narayan, R. 1997, ApJ, 489, 865

Fabian, A. C., Guilbert, P. W., Motch, C., Ricketts, M., Ilovaisky, S. A., \& Chevalier, C. 1982, A\&A, 111, L9

Ghisellini, G., Guilbert, P. W., \& Svensson, R. 1988, ApJ, 334, L5

Ghisellini, G., Haardt, F., \& Svensson, R. 1998, MNRAS, 297, 348

Gierlinski, M., Zdziarski, A. A., Done, C., Johnson, W. N., Ebisawa, K., Ueda, Y., Haardt, F., \& Phlips, B. F. 1997, MNRAS, 288, 958

Gierliński, M., Zdziarski, A. A., Poutanen, J., Coppi, P. S., Ebisawa, K., \& Johnson, W. N. 1999, MNRAS, 309, 496

Grove, J. E., Johnson, W. N., Kroeger, R. A., McNaron-Brown, K., Skibo, J. G., \& Phlips, B. F. 1998, ApJ, 500, 899

Haardt, F. \& Maraschi, L. 1993, ApJ, 413, 507

Haardt, F., Maraschi, L., \& Ghisellini, G. 1994, ApJ, 432, L95

Kanbach, G., Straubmeier, C., Spruit, H. C., \& Belloni, T. 2001, Nature, 414,180

Katarzyński, K., Ghisellini, G., Svensson, R., \& Gracia, J. 2006, A\&A, 451, 739

Ling, J. C., et al. 1997, ApJ, 484, 375
McConnell, M., et al. 1994, ApJ, 424, 933

McConnell, M. L., et al. 2002, ApJ, 572, 984

Magdziarz, P. \& Zdziarski, A. A. 1995, MNRAS, 273, 837

Malzac, J., Beloborodov, A. M., \& Poutanen, J. 2001, MNRAS, 326, 417

Motch, C. Ilovaisky, S. A., \& Chevalier, C. 1982, A\&A, 109, L1

Narayan, R. \& Yi, I. 1995, ApJ, 452, 710

Narayan, R., Mahadevan, R., \& Quataert, E. 1998, in Theory of Black Hole Accretion Disks, ed. M. A. Abramowicz, G. Björnsson, J. E. Pringle

(Cambridge: Cambridge Univ. Press), 148

Poutanen, J. 1998, in Theory of Black Hole Accretion Disks, ed. M. A

Abramowicz, G. Björnsson, J. E. Pringle (Cambridge: Cambridge Univ. Press), 100

Poutanen, J. \& Coppi, P. S. 1998, Phys. Scr., T77, 57

Poutanen, J., Krolik, J. H., \& Ryde, F. 1997, MNRAS, 292, L21

Rees, M. J. 1967, MNRAS, 136, 279

Stern, B. E., Poutanen, J., Svensson, R., Sikora, M., \& Begelman, M. C. 1995, ApJ, 449, L13

Svensson, R. 1999, in ASP Conf. Ser. 161, High Energy Processes in

Accreting Black Holes, ed. J. Poutanen \& R. Svensson (San Francisco: ASP), 361

Vurm, I. \& Poutanen, J. 2008a, Int. J. Mod. Physics, 17, 1629

—. 2008b, ApJ, submitted (arXiv:0807.2540)

Wardziński, G. \& Zdziarski, A. A. 2001, MNRAS, 325, 963

Yuan, F. 2003, ApJ, 594, L99

Yuan, F. \& Zdziarski, A. A. 2004, MNRAS, 354, 953 
Zdziarski, A. A. \& Gierliński, M. 2004, Prog. Theor. Phys. Suppl., 155, 99 Zdziarski, A. A., Grove, J. E., Poutanen, J., Rao, A. R., \& Vadawale, S. V. 2001, ApJ, 554, L45

Zdziarski, A. A., Johnson, W. N., Poutanen, J., Magdziarz, P., \& Gierlinski, M. 1997, in ESA SP-382, The Transparent Universe, ed. C. Winkler,

T. J.-L. Courvoisier, \& P. Durouchoux (Noordwijk: ESA), 373
Zdziarski, A. A., Lubinski, P., \& Smith, D. A. 1999, MNRAS, 303, L11 Zdziarski, A. A., Poutanen, J., Paciesas, W. S., \& Wen, L. 2002, ApJ, 578, 357 